\title{
Research Article Circular Slits Map of Bounded Multiply Connected Regions
}

\author{
Ali W. K. Sangawi, ${ }^{1,2,3}$ Ali H. M. Murid, ${ }^{1,2}$ and M. M. S. Nasser ${ }^{4,5}$ \\ ${ }^{1}$ Ibnu Sina Institute for Fundamental Science Studies, Universiti Teknologi Malaysia, Johor, \\ 81310 Johor Bahru, Malaysia \\ 2 Department of Mathematics, Faculty of Science, Universiti Teknologi Malaysia, Johor, \\ 81310 Johor Bahru, Malaysia \\ ${ }^{3}$ Department of Mathematics, School of Science, University of Sulaimani, \\ Sulaimani 46001, Iraq \\ ${ }^{4}$ Department of Mathematics, Faculty of Science, Ibb University, P.O. Box 70270, Ibb, Yemen \\ ${ }^{5}$ Department of Mathematics, Faculty of Science, King Khalid University, P.O. Box 9004, \\ Abha, Saudi Arabia \\ Correspondence should be addressed to Ali H. M. Murid, alihassan@utm.my
}

Received 14 December 2011; Revised 25 January 2012; Accepted 26 January 2012

Academic Editor: Karl Joachim Wirths

Copyright (C) 2012 Ali W. K. Sangawi et al. This is an open access article distributed under the Creative Commons Attribution License, which permits unrestricted use, distribution, and reproduction in any medium, provided the original work is properly cited.

We present a boundary integral equation method for the numerical conformal mapping of bounded multiply connected region onto a circular slit region. The method is based on some uniquely solvable boundary integral equations with adjoint classical, adjoint generalized, and modified Neumann kernels. These boundary integral equations are constructed from a boundary relationship satisfied by a function analytic on a multiply connected region. Some numerical examples are presented to illustrate the efficiency of the presented method.

\section{Introduction}

In general, the exact conformal mapping functions are unknown except for some special regions. It is well known that every multiply connected regions can be mapped conformally onto the circle with concentric circular slits, the circular ring with concentric circular slits, the circular slit region, the radial slit region, and the parallel slit region as described in Nehari [1, page 334]. Several methods for numerical approximation for the conformal mapping of multiply connected regions have been proposed in [2-16]. Recently, reformulations of conformal mappings from bounded and unbounded multiply connected regions onto the five 
canonical slit regions as Riemann-Hilbert problems are discussed in Nasser [12, 13, 17]. An integral equation with the generalized Neumann kernel is then used to solve the RH problem as developed in [18]. The integral equation however involves singular integral which is calculated by Wittich's method. Murid and Hu [11] formulated an integral equation method based on another form of generalized Neumann kernel for conformal mapping of bounded doubly connected regions onto a disk with circular slit but the kernel of the integral equation involved the unknown circular radii. Discretization of the integral equation yields a system of nonlinear equations which they solved using an optimization method. To overcome this nonlinear problem, Sangawi et al. [19] have developed linear integral equations for conformal mapping of bounded multiply connected regions onto a disk with circular slits. In this paper, we describe an integral equation method for computing the conformal mapping function $f$ of bounded multiply connected regions onto a circular slit region. This boundary integral equation is constructed from a boundary relationship that relates the mapping function $f$ on a multiply connected region with $f^{\prime}, \theta^{\prime}(t)$, and $|f|$, where $\theta$ is the boundary correspondence function.

The plan of the paper is as follows. Section 2 presents some auxiliary materials. Derivations of two integral equations related to $f^{\prime}$ and $\theta^{\prime}(t)$ are given in Sections 3 and 4, respectively. Section 5 presents a method to calculate the modulus of $f$. In Section 6 , we give some examples to illustrate our boundary integral equation method. Finally, Section 7 presents a short conclusion.

\section{Notations and Auxiliary Material}

Let $\Omega$ be a bounded multiply connected region of connectivity $M+1$. The boundary $\Gamma$ consists of $M+1$ smooth Jordan curves $\Gamma_{j}, j=0,1, \ldots, M$, such that $\Gamma_{\hat{j}}, \widehat{j}=1, \ldots, M$, lies in the interior of $\Gamma_{0}$, where the outer curve $\Gamma_{0}$ has counterclockwise orientation and the inner curves $\Gamma_{\hat{j}}$, $\widehat{j}=1, \ldots, M$, have clockwise orientation. The positive direction of the contour $\Gamma=\bigcup_{j=0}^{M} \Gamma_{j}$ is usually that for which $\Omega$ is on the left as one traces the boundary (see Figure 1). The curve $\Gamma_{k}$ is parametrized by $2 \pi$-periodic twice continuously differentiable complex function $z_{k}(t)$ with nonvanishing first derivative

$$
z_{k}^{\prime}(t)=\frac{d z_{k}(t)}{d t} \neq 0, \quad t \in J_{k}=[0,2 \pi], k=0,1, \ldots, M .
$$

The total parameter domain $J$ is the disjoint union of $M+1$ intervals $J_{0}, \ldots, J_{M}$. We define a parametrization $z$ of the whole boundary $\Gamma$ on $J$ by

$$
z(t)=\left\{\begin{array}{l}
z_{0}(t), \quad t \in J_{0}=[0,2 \pi], \\
\vdots \\
z_{M}(t), \quad t \in J_{M}=[0,2 \pi] .
\end{array}\right.
$$



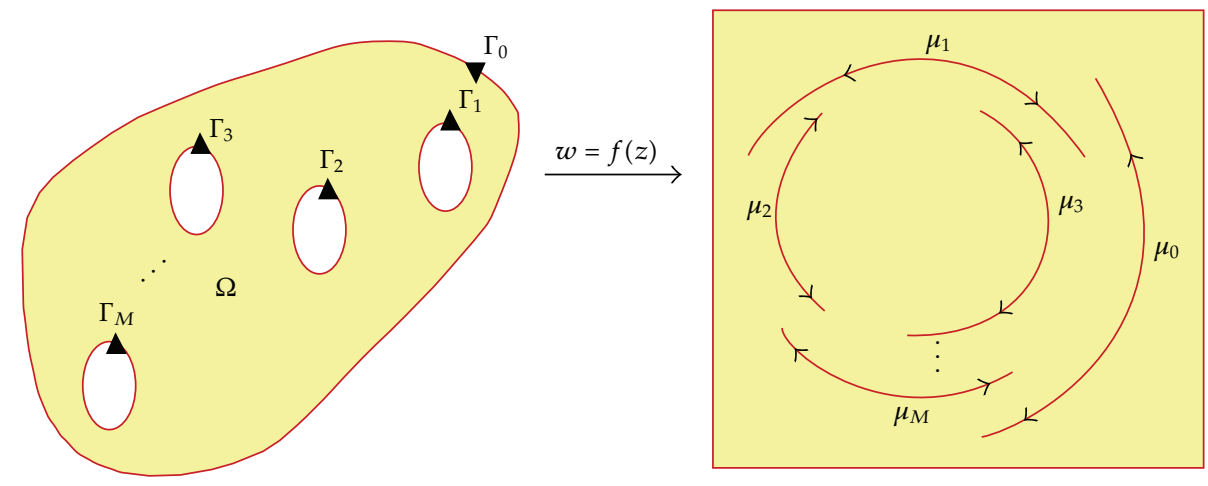

Figure 1: Mapping of the bounded multiply connected region $\Omega$ of connectivity $M+1$ onto a circular slit region.

Let $H^{*}$ be the space of all real Hölder continuous $2 \pi$-periodic functions $\omega(t)$ of the parameter $t$ on $J_{k}$ for $k=0,1, \ldots, M$, that is,

$$
\omega(t)= \begin{cases}\omega_{0}(t), & t \in J_{0}, \\ \omega_{1}(t), & t \in J_{1}, \\ \vdots & \\ \omega_{M}(t), & t \in J_{M} .\end{cases}
$$

Let $\theta(t)$ (the boundary corresponding function) be given for $t \in J$ by

$$
\theta(t)= \begin{cases}\theta_{0}(t), & t \in J_{0} \\ \vdots & \\ \theta_{M}(t), & t \in J_{M} .\end{cases}
$$

Let $\mu$ (a piecewise constant real function) be given for $t \in J$ by

$$
\mu(t)=\left(\mu_{0}, \mu_{1}, \ldots, \mu_{M}\right)= \begin{cases}\mu_{0}, & t \in J_{0}, \\ \vdots & \\ \mu_{M}, & t \in J_{M} .\end{cases}
$$

Let $\widehat{A}(t)$ be a complex continuously differentiable $2 \pi$-periodic function for all $t \in J$. The generalized Neumann kernel formed with $\widehat{A}$ is defined by

$$
\widehat{N}(t, s)=\frac{1}{\pi} \operatorname{Im}\left(\frac{\widehat{A}(t)}{\widehat{A}(s)} \frac{z^{\prime}(s)}{z(s)-z(t)}\right) .
$$


The kernel $\widehat{N}$ is continuous with

$$
\widehat{\mathrm{N}}(t, t)=\frac{1}{\pi}\left(\frac{1}{2} \operatorname{Im} \frac{z^{\prime \prime}(t)}{z^{\prime}(t)}-\operatorname{Im} \frac{\widehat{A}^{\prime}(t)}{\widehat{A}(t)}\right) .
$$

Define also the kernel $\widehat{M}$ by

$$
\widehat{M}(t, s)=\frac{1}{\pi} \operatorname{Re}\left(\frac{\widehat{A}(t)}{\widehat{A}(s)} \frac{z^{\prime}(s)}{z(s)-z(t)}\right),
$$

which has a cotangent singularity type (see [18] for more detail). The classical Neumann kernel is the generalized Neumann kernel formed with $\widehat{A}(t)=1$, that is,

$$
N(t, s)=\frac{1}{\pi} \operatorname{Im}\left(\frac{z^{\prime}(s)}{z(s)-z(t)}\right) .
$$

The adjoint kernel $N^{*}(s, t)$ of the classical Neumann kernel is given by

$$
N^{*}(t, s)=N(s, t)=\frac{1}{\pi} \operatorname{Im}\left(\frac{z^{\prime}(t)}{z(t)-z(s)}\right) .
$$

The adjoint function to the function $\widehat{A}$ is given by

$$
\tilde{A}(t)=\frac{z^{\prime}(t)}{\widehat{A}(t)}=z^{\prime}(t) .
$$

The generalized Neumann kernel $\widetilde{N}(s, t)$ formed with $\widetilde{A}$ is given by

$$
\widetilde{N}(t, s)=\frac{1}{\pi} \operatorname{Im}\left(\frac{\tilde{A}(t)}{\tilde{A}(s)} \frac{z^{\prime}(s)}{z(s)-z(t)}\right) .
$$

If $\widehat{A}=1$, then

$$
\widetilde{N}(t, s)=-N^{*}(t, s)
$$


We define the Fredholm integral operators $\mathbf{N}, \tilde{\mathbf{N}}, \mathbf{N}^{*}$ by

$$
\begin{aligned}
& \mathbf{N} v(t)=\int_{J} N(t, s) v(s) d s, \quad t \in J, \\
& \tilde{\mathbf{N}} v(t)=\int_{J} \widetilde{N}(t, s) v(s) d s, \quad t \in J, \\
& \mathbf{N}^{*} v(t)=\int_{J} N(s, t) v(s) d s, \quad t \in J .
\end{aligned}
$$

Note that $\tilde{\mathbf{N}}=-\mathbf{N}^{*}$, if $\widehat{A}=1$.

It is known that $\lambda=1$ is an eigenvalue of the kernel $N$ with multiplicity 1 and $\lambda=-1$ is an eigenvalue of the kernel $N$ with multiplicity $M$ [18]. We define the piecewise constant functions

$$
x^{[j]}(\xi)= \begin{cases}1, & \xi \in \Gamma_{j}, j=0,1,2, \ldots, M \\ 0, & \text { otherwise. }\end{cases}
$$

Then, we have from [18]

$$
\operatorname{Null}(\mathbf{I}-\mathbf{N})=\operatorname{span}\{1\}, \quad \operatorname{Null}(\mathbf{I}-\mathbf{N})=\operatorname{span}\left\{x^{[1]}, \chi^{[2]}, \ldots, \chi^{[M]}\right\}
$$

Lastly, we define integral operators $\mathbf{J}$ and $\widehat{\mathbf{J}}$ by

$$
\begin{aligned}
& \mathbf{J} v=\int_{J} \frac{1}{2 \pi} \sum_{j=1}^{M} X^{[j]}(s) X^{[j]}(t) v(s) d s, \\
& \widehat{\mathbf{J}} v=\int_{J} \frac{1}{2 \pi} \sum_{j=0}^{M} X^{[j]}(s) X^{[j]}(t) v(s) d s,
\end{aligned}
$$

which are required for uniqueness of solution in a later section.

\section{Homogenous and Nonhomogenous Boundary Relationship}

\subsection{Nonhomogeneous Boundary Relationship for Conformal Mapping}

Suppose that $c(z), Q(z)$, and $H(z)$ are complex-valued functions defined on $\Gamma$ such that $c(z) \neq 0, H(z) \neq 0, Q(z) \neq 0$, and $\overline{H(z)} /(T(z) Q(z))$ satisfies the Hölder condition on $\Gamma$. Then, the interior relationship is defined as follows.

A complex-valued function $P(z)$ is said to satisfy the interior relationship if $P(z)$ is analytic in $\Omega$ and satisfies the nonhomogeneous boundary relationship

$$
P(z)=c(z) \frac{\overline{T(z) Q(z)}}{\overline{G(z)}} \overline{P(z)}+\overline{H(z)}, \quad z \in \Gamma,
$$


where $G(z)$ analytic in $\Omega$, Hölder continuous on $\Gamma$, and $G(z) \neq 0$ on $\Gamma$. The boundary relationship (3.1) also has the following equivalent form:

$$
G(z)=\overline{c(z)} T(z) Q(z) \frac{P(z)^{2}}{|P(z)|^{2}}+\frac{G(z) H(z)}{\overline{P(z)}}, \quad z \in \Gamma
$$

Let the function $L_{R}(\tilde{z})$ be defined in the region $C \cup\{\infty\} \backslash \Gamma$ by

$$
L_{R}(\tilde{z})=\frac{1}{2 \pi \mathrm{i}} \int_{\Gamma} \frac{\overline{c(\widetilde{z})} H(w)}{\overline{c(w)}(w-\tilde{z}) Q(w) T(w)} d w, \quad \tilde{z} \in \Omega^{-}
$$

where $\Omega^{-}$is the complement of $\Omega$. The following theorem gives an integral equation for an analytic function satisfying the interior nonhomogeneous boundary relationship (3.1) or (3.2). This theorem generalizes the results of Murid and Razali [9] and can be proved by using the approach used in proving Theorem 3.1 in [20, page 45].

Theorem 3.1. Let $U$ and $V$ be any complex-valued functions that are defined on $\Gamma$. If the function $P(z)$ satisfies the interior nonhomogeneous boundary relationship (3.1) or (3.2), then

$$
\begin{gathered}
\frac{1}{2}\left[V(z)+\frac{U(z)}{\overline{T(z) Q(z)}}\right] P(z)+P V \int_{\Gamma} K(z, w) P(w)|d w|+c(z) U(z) \\
\times\left[\sum_{a_{j} \in \Omega^{2} w=a_{j}} \frac{P(w)}{(w-z) G(w)}\right]^{\mathrm{conj}}=-U(z) \overline{L_{R}^{-}(z)}, \quad z \in \Gamma,
\end{gathered}
$$

where

$$
\begin{gathered}
K(z, w)=\frac{1}{2 \pi i}\left[\frac{c(z) U(z)}{c(w)(\bar{w}-\bar{z}) \overline{Q(w)}}-\frac{V(z) T(w)}{w-z}\right] \\
L_{R}^{-}(z)=\frac{-1}{2} \frac{H(z)}{Q(z) T(z)}+\mathrm{PV} \frac{1}{2 \pi i} \int_{\Gamma} \frac{c(w)}{\overline{c(w)}(w-z) Q(w) T(w)} d w .
\end{gathered}
$$

The symbol "conj" in the superscript denotes complex conjugate, while the minus sign in the superscript denotes limit from the exterior. The sum in (3.4) is over all those zeros $a_{1}, a_{2}, \ldots, a_{M}$ of $G$ that lie inside $\Omega$. If $G$ has no zeros in $\Omega$, then the term containing the residue in (3.4) will not appear.

Proof. Suppose that $P(z)$ and $G(z)$ are analytic functions in $\Omega$ and $G$ has a finite number of zeros at $a_{1}, a_{2}, \ldots, a_{M}$ in $\Omega$. Then, by the calculus of residues, we have

$$
\frac{1}{2 \pi i} \int_{\Gamma} \frac{P(w)}{(w-\tilde{z}) G(w)} d w=\sum_{a_{j} \in \Omega} \operatorname{Res}_{w=a_{j}} \frac{P(w)}{(w-\tilde{z}) G(w)}, \quad \tilde{z} \in \Omega^{-}
$$


Since $P$ and $G$ satisfy the Hölder condition on $\Gamma$ and $G(z) \neq 0$ on $\Gamma$, then $P / G$ also satisfies the Hölder condition on $\Gamma$. Taking the limit $\Omega^{-} \ni \widetilde{z} \rightarrow z \in \Gamma$ and applying Sokhotski formula [5], we get

$$
-\frac{1}{2} \frac{P(z)}{G(z)}+\operatorname{PV} \frac{1}{2 \pi i} \int_{\Gamma} \frac{P(z)}{(w-z) G(w)} d w=\sum_{a_{j} \in \Omega} \operatorname{Res}_{w=a_{j}} \frac{P(w)}{(w-z) G(w)}, \quad z \in \Gamma .
$$

By taking conjugate to both sides and using (3.1), we get

$$
\begin{aligned}
& -\frac{1}{2} \frac{P(z)}{c(z) \overline{Q(z) \mathrm{T}(z)}}+\frac{1}{2} \frac{\overline{H(z)}}{c(z) \overline{Q(z) T(z)}}-\mathrm{PV} \frac{1}{2 \pi i} \int_{\Gamma} \frac{P(z)}{c(w)(\bar{w}-\bar{z}) \overline{Q(w)}} \frac{\overline{d w}}{\overline{T(w)}} \\
& +\operatorname{PV} \frac{1}{2 \pi i} \int_{\Gamma} \frac{\overline{H(z) d w}}{c(w)(\bar{w}-\bar{z}) \overline{Q(z) T(z)}}=\left[\sum_{a_{j} \in \Omega} \operatorname{Res}_{w=a_{j}} \frac{P(w)}{(w-z) G(w)}\right]^{\text {conj }}, \quad z \in \Gamma .
\end{aligned}
$$

Multiplying both sides by $-c(z)$ and the fact that $d w=T(w)|d w|$, after some arrangement, yield

$$
\begin{gathered}
\frac{1}{2} \frac{P(z)}{\overline{Q(z) T(z)}}+P V \frac{1}{2 \pi i} \int_{\Gamma} \frac{c(z) P(z)}{c(w)(\bar{w}-\bar{z}) \overline{Q(w)}}|d w|+c(z)\left[\sum_{a_{j} \in \Omega} \operatorname{Res}_{w=a_{j}} \frac{P(w)}{(w-z) G(w)}\right]^{\text {conj }} \\
=-\left[-\frac{1}{2} \frac{H(z)}{Q(z) T(z)}+\operatorname{PV} \frac{1}{2 \pi i} \int_{\Gamma} \frac{\overline{c(z)} H(z)}{\overline{c(w)}(w-z) Q(z) T(z)} d w\right]^{\text {conj }}, \quad z \in \Gamma .
\end{gathered}
$$

Applying Sokhotski formulas again to the expression inside the bracket of the right-hand side yields

$$
\begin{aligned}
& \frac{1}{2} \frac{P(z)}{\overline{Q(z) T(z)}}+P V \frac{1}{2 \pi i} \int_{\Gamma} \frac{c(z) P(z)}{c(w)(\bar{w}-\bar{z}) \overline{Q(w)}}|d w|+c(z)\left[\sum_{a_{j} \in \Omega} \operatorname{Res}_{w=a_{j}} \frac{P(w)}{(w-z) G(w)}\right]^{\text {conj }} \\
& =-\overline{L_{R}^{-}(z)}, \quad z \in \Gamma .
\end{aligned}
$$

Since $P(z)$ is analytic in $\Omega$, then by Cauchy integral formula, we have

$$
\frac{1}{2 \pi i} \int_{\Gamma} \frac{P(z)}{w-\widetilde{z}} d w=0, \quad z \in \Omega^{-}
$$

Taking the limit $\omega^{-} \ni \tilde{z} \rightarrow z \in \Gamma$ and applying Sokhotiski formulas, we get

$$
-\frac{1}{2} P(z)+P V \frac{1}{2 \pi i} \int_{\Gamma} \frac{T(w) P(z)}{w-z}|d w|=0, \quad z \in \Gamma
$$

Multiplying (3.12) by $v(z)$ and subtracting it from (3.10) multiplied by $u(z)$ yield (3.4). 


\subsection{Homogeneous Boundary Relationship for Conformal Mapping}

Let $w=f(z)$ be the analytic function which maps $\Omega$ in the $z$-plane onto a canonical region of the circular slit region in the $w$-plane. Let 0 and $a$ be a fixed point in $\Omega$ such that $a \neq 0$. Then, the mapping function is made uniquely determined by assuming that $f(a)=0$ and $f(0)=\infty$ such that the residue of the function $f$ at 0 is equal to 1 [1]. Hence, the function $f$ can be written in the form

$$
f(z)=\left(\frac{1}{z}-\frac{1}{a}\right) e^{z g(z)}
$$

where $g$ is analytic in $\Omega[12,13]$. Note that the boundary value of $f$ can be represented in the form

$$
f\left(z_{p}(t)\right)=\mu_{p} e^{i \theta_{p}(t)}, \quad \Gamma_{p}: z=z_{p}(t), \quad 0 \leq t \leq \beta_{p}, p=0,1, \ldots, M,
$$

where $\theta_{p}$ is a boundary correspondence function of $\Gamma_{p}$ and $\mu_{p}$ is the radius of the circular slit. The unit tangent to $\Gamma$ at $z(t)$ is denoted by $T(z(t))=z^{\prime}(t) /\left|z^{\prime}(t)\right|$. Thus, it can be shown that

$$
f(z)=\frac{|f(z)|}{i} T(z) \frac{\left|\theta_{p}^{\prime}(t)\right|}{\theta_{p}^{\prime}(t)} \frac{f^{\prime}(z)}{\left|f^{\prime}(z)\right|}, \quad z \in \Gamma .
$$

\section{Integral Equation Method for Computing $F^{\prime}(Z)$}

Note that the value of $\theta_{p}^{\prime}(t)$ may be positive or negative since each circular slit $f\left(\Gamma_{p}\right)$ is traversed twice. Thus, $\left|\theta_{p}^{\prime}\right| / \theta_{p}^{\prime}= \pm 1$. Hence, the boundary relationship (3.15) can be written as

$$
f(z)= \pm T(z) \frac{|f(z)|}{i} \frac{f^{\prime}(z)}{\left|f^{\prime}(z)\right|}, \quad z \in \Gamma
$$

To eliminate the \pm sign, we square both sides of the boundary relationship (4.1) to get

$$
f(z)^{2}=-T(z)^{2}|f(z)|^{2} \frac{f^{\prime}(z)^{2}}{\left|f^{\prime}(z)\right|^{2}}, \quad z \in \Gamma .
$$

Then, the function $E(z)$ defined by

$$
D(z)=z^{2} f^{\prime}(z)=z^{2} f(z)\left[z g^{\prime}(z)+g(z)\right]-e^{z g(z)}
$$

is analytic in $\Omega$.

Combining (4.3), (4.2), and (3.13), we obtain the following boundary relationship:

$$
\frac{z e^{2 z h(z)}}{a^{2}}=-\frac{\bar{z}|z|^{2}}{(a-z)^{2}}|f(z)|^{2} T(z)^{2} \frac{D(z)^{2}}{|D(z)|^{2}}, \quad z \in \Gamma .
$$


Comparison of (4.4) and (3.2) leads to a choice of $P(z)=D(z), c(z)=-z|z|^{2}|f(z)|^{2} /(\bar{a}-\bar{z})^{2}$, $Q(z)=T(z), G(z)=z e^{2 z h(z)} / a^{2}, H(z)=0$. Setting $U(z)=\overline{T(z) Q(z)}$ and $V(z)=1$, Theorem 3.1 yields

$$
\begin{gathered}
T(z) D(z)+\operatorname{PV} \frac{1}{2 \pi \mathrm{i}} \int_{\Gamma}\left[\frac{z|z|^{2}|f(z)|^{2} \overline{(a-w)^{2}} \overline{T(z)}}{w|w|^{2}|f(w)|^{2} \overline{(a-z)^{2}}(\bar{w}-\bar{z})}-\frac{T(z)}{w-z}\right] T(w) D(w)|d w| \\
=\frac{z|z|^{2}|f(z)|^{2}}{(\bar{a}-\bar{z})^{2}} \overline{T(z)}\left[\sum_{a_{j} \in \Omega^{w=a_{j}}} \operatorname{Res}_{(w-z) w e^{2 w h(w)}}\right]^{\text {conj }}, \quad z \in \Gamma .
\end{gathered}
$$

Note that $a^{2} D(w) /(w-z) w^{2}$ has a simple pole at $w=0$. To evaluate the residue in (4.5), we use the fact that if $L(z)=d(z) / q(z)$ where $d(z)$ and $q(z)$ are analytic at $z_{0}$ and $d\left(z_{0}\right) \neq 0$, $q\left(z_{0}\right)=0$ and $q^{\prime}\left(z_{0}\right) \neq 0$, which means $z_{0}$ is a simple pole of $L(z)$, then

$$
\operatorname{Res}_{w=z_{0}} L(w)=\frac{d\left(z_{0}\right)}{q^{\prime}\left(z_{0}\right)}
$$

Applying (4.6) to the residue in (4.5) and after several algebraic manipulations, we obtain

$$
\sum_{a_{j} \in \Omega} \operatorname{Res}_{w=a_{j}} \frac{a^{2} D(w)}{(w-z) w e^{2 w g(w)}}=\frac{a^{2}}{z}
$$

Thus, integral equation (4.5) becomes

$$
F(Z)+\int_{\Gamma} N^{+}(z, w) F(w)|d w|=\frac{\overline{a^{2}} z^{2}|f(z)|^{2}}{(\bar{a}-\bar{z})^{2}} \overline{T(z)}, \quad z \in \Gamma,
$$

where

$$
\begin{aligned}
F(z) & =T(z) D(z), \\
D(z) & =z^{2} f^{\prime}(z), \\
N^{+}(z, w) & =\frac{1}{2 \pi i}\left[\frac{T(z)}{z-w}-\frac{z|z|^{2}|f(z)|^{2} \overline{(a-w)^{2}} \overline{T(z)}}{w|w|^{2}|f(w)|^{2} \overline{(a-z)^{2}}(\bar{z}-\bar{w})}\right], \\
N^{+}(t, t) & =\frac{1}{2 \pi\left|z^{\prime}(t)\right|} \operatorname{Im} \frac{z^{\prime \prime}(t)}{z^{\prime}(t)}+\frac{1}{\pi i\left|z^{\prime}(t)\right|}\left[\frac{\overline{z^{\prime}(t)}}{\overline{z(t)}-\bar{a}}-\operatorname{Re}\left(\frac{z^{\prime}(t)}{z(t)}\right)\right] \\
& -\frac{1}{2 \pi i\left|z^{\prime}(t)\right|} \frac{z^{\prime}(t)}{z(t)} .
\end{aligned}
$$


By using single valuedness of the mapping function $f$ leads to the following condition:

$$
\frac{1}{2 \pi} \int_{-\Gamma_{q}} \frac{F(w)}{w^{2}}|d w|=0, \quad q=0,1, \ldots, M
$$

By means of Cauchy's integral formula, we can get the following condition:

$$
\frac{1}{2 \pi} \int_{\Gamma} \frac{F(w)}{w}|d w|=-i
$$

Thus, the integral equation (4.8) with the conditions (4.10) and (4.11) should give a unique solution provided the parameters $\mu_{p}, p=0,1, \ldots, M$ that appear in $N^{+}(z, w)$ are known. sections.

Integral equation methods for computing $\mu_{p}$ and $\theta_{p}^{\prime}$ are discussed in the next two

\section{Integral Equation for Computing $|f(z)|$}

Note that, from (3.13) and (3.14), we get the following equation:

$$
z(t) g(z(t))=\log |f(z(t))|-\log \left|\frac{1}{z(t)}-\frac{1}{a}\right|-i \arg \left(\frac{1}{z(t)}-\frac{1}{a}\right)+\theta_{p}(t) .
$$

Since $g(z)$ is analytic in $\Omega$, thus

$$
\widehat{A}(t) g(z(t))=\gamma(t)+h(t)+i v,
$$

from (5.1) and (5.2), yields

$$
\begin{gathered}
\hat{A}(t)=z(t), \\
\gamma(t)=-\log \left(\frac{1}{z(t)}-\frac{1}{a}\right), \\
h(t)=\log \mu(t)=\left(\log \mu_{0}, \log \mu_{1}, \ldots, \log \mu_{M}\right) .
\end{gathered}
$$
$\left|f\left(z_{p}\right)\right|$.

The following theorem from [22] gives a method for calculating $h(t)$, and hence $\mu_{p}=$

Theorem 5.1 (see [22, Theorem 5]). The function $h$ is given by $h=\left(h_{0}, h_{1}, \ldots, h_{M}\right)$, where

$$
h_{j}=\left(\gamma, \phi^{[j]}\right)=\frac{1}{2 \pi} \int_{J} \gamma(t) \phi^{[j]}(t) d t
$$

and where $\phi^{[j]}$ is the unique solution of the following integral equation

$$
\left(\mathbf{I}+\widehat{\mathbf{N}}^{*}+\widehat{\mathbf{J}}\right) \phi^{[j]}=-\chi^{[j]}, \quad j=0,1, \ldots, M,
$$

where the kernel $\widehat{N}^{*}(s, t)$ is the adjoint kernel of the kernel $\widehat{N}(s, t)$ which is formed with $\widehat{A}(t)=z(t)$. 
By obtaining $h_{0}, h_{1}, \ldots, h_{M}$ from (5.6), in view of (5.5), we obtain

$$
\mu_{j}=e^{h_{j}}, \quad j=0,1, \ldots, M
$$

\section{Integral Equation Method for Computing $\theta_{p}^{\prime}(t)$}

This section gives another application of Theorem 3.1 for computing $f^{\prime} / f$. Let $f$ be the mapping function as described in Section 3.2. Note that (4.2) can be written in the following form:

$$
\left|\frac{f^{\prime}(z)}{\mathrm{f}(z)}\right|^{2}=-T(z)^{2}\left(\frac{f^{\prime}(z)}{f(z)}\right)^{2}, \quad z \in \Gamma
$$

Taking the derivative of both sides of (3.13) together with some elementary calculations yields

$$
\frac{f^{\prime}(z)}{f(z)}+\frac{a}{z(a-z)}=z g^{\prime}(z)+g(z)
$$

Let $E(z)=\left(f^{\prime}(z) / f(z)\right)+(a / z(a-z))=z g^{\prime}(z)+g(z)$ be analytic in $\Omega$. Then,

$$
\frac{f^{\prime}(z)}{f(z)}=E(z)+\frac{a}{z(z-a)}, \quad z \in \Gamma
$$

Equations (6.1) and (6.3) together with some elementary calculations yield

$$
E(z)=-\overline{T(z)^{2}} \overline{E(z)}-\frac{\bar{a} \overline{T(z)^{2}}}{\bar{z}(\bar{z}-\bar{a})}-\frac{a}{z(z-a)}, \quad z \in \Gamma .
$$

Comparison of (6.4) and (3.1) leads to a choice of $P(z)=E(z), c(z)=-1, Q(z)=T(z)$, $G(z)=1, H(z)=-\left(a T(z)^{2} / z(z-a)\right)-(\bar{a} / \bar{z}(\bar{z}-\bar{a}))$. Setting $U(z)=\overline{T(z) Q(z)}$ and $V(z)=1$, Theorem 3.1 yields

$$
E(z) T(z)+\operatorname{PV} \frac{1}{2 \pi i} \int_{\Gamma}\left[\frac{\overline{T(z)}}{\bar{w}-\bar{z}}-\frac{T(z)}{w-z}\right] E(w) T(w)|d w|=-\overline{T(z)} \overline{L_{R}^{-}(z)}, \quad z \in \Gamma,
$$

where

$$
\begin{aligned}
T(z) L_{R}^{-}(z)= & -\frac{1}{2}\left[\frac{-a T(z)}{z(z-a)}-\frac{\bar{a} \overline{T(z)}}{\bar{z}(\bar{z}-\bar{a})}\right]+T(z) \operatorname{PV} \frac{1}{2 \pi i} \int_{\Gamma} \frac{a}{w(w-z)(w-a)} d w \\
& -T(z) \operatorname{PV} \frac{1}{2 \pi i} \int_{\Gamma} \frac{A \bar{a} \overline{T(w)^{2}}}{\bar{w}(\bar{w}-\bar{a})(w-z)} d w, \quad z \in \Gamma .
\end{aligned}
$$


Then, it follows from [5, page 91] that

$$
\mathrm{PV} \frac{1}{2 \pi i} \int_{\Gamma} \frac{a}{w(w-z)(w-a)} d w=-\frac{1}{2} \frac{a}{z(z-a)}
$$

From (6.5),(6.6), (6.7), and (6.3), we obtain the integral equation

$$
\frac{f^{\prime}(z)}{f(z)} T(z)+\operatorname{PV} \frac{1}{2 \pi i} \int_{\Gamma}\left[\frac{T(z)}{z-w}-\frac{\overline{T(z)}}{\bar{z}-\bar{w}}\right] \frac{f^{\prime}(w)}{f(w)} T(w)|d w|=2 i \operatorname{Im}\left[\frac{a T(z)}{z(z-a)}\right], \quad z \in \Gamma .
$$

In the above integral equation, let $z=z(t)$ and $w=z(s)$. Then, by multiplying both sides of (6.8) by $\left|z^{\prime}(t)\right|$ and using the fact that

$$
\frac{f^{\prime}(z)}{f(z)} z^{\prime}(t)=i \theta_{p}^{\prime}(t), \quad z \in \Gamma
$$

the above integral equation can also be written as

$$
\theta_{p}^{\prime}(t)+\int_{J} N(s, t) \theta_{p}^{\prime}(s) d s=2 \operatorname{Im}\left[\frac{a z^{\prime}(t)}{z(t)(z(t)-a)}\right]
$$

Since $N(s, t)=N^{*}(t, s)$, the integral equation can be written as an integral equation in operator form

$$
\left(\mathbf{I}+\mathbf{N}^{*}\right) \theta_{p}^{\prime}=\tilde{\psi},
$$

where

$$
\widetilde{\psi}=2 \operatorname{Im}\left[\frac{a z^{\prime}(t)}{z(t)(z(t)-a)}\right]
$$

However, $\lambda=-1$ is an eigenvalue of $N^{*}$ with multiplicity $M$, by [18, Theorem 12]. Therefore, the integral equation (6.11) is not uniquely solvable. To overcome this problem, note that

$$
\int_{J_{j}} \theta_{p}^{\prime}(t) d t=0, \quad j=1,2, \ldots, M
$$

which implies

$$
\mathrm{J} \theta_{p}^{\prime}=0
$$

By adding (6.14) to (6.11), we obtain the integral equation

$$
\left(\mathbf{I}+\mathbf{N}^{*}+\mathbf{J}\right) \theta_{p}^{\prime}=\tilde{\psi}
$$


The integral equation (6.15) is uniquely solvable in view of the following theorem which can be proved by using the approach used in proving [22, Theorem 4].

Theorem 6.1.

$$
\operatorname{Null}\left(\mathbf{I}+\mathbf{N}^{*}+\mathbf{J}\right)=\{0\}
$$

Proof. Let $v \in \operatorname{Null}\left(\mathbf{I}+\mathbf{N}^{*}+\mathbf{J}\right)$, that is, $v$ is a solution of the integral equation

$$
\left(\mathbf{I}+\mathbf{N}^{*}+\mathbf{J}\right) v=0
$$

Then, it follows from the definition of the operator $\mathbf{J}$, (2.18), and the Fredholm alternative theorem that

$$
\begin{gathered}
\mathbf{J}=\mathbf{J}^{*}=\mathbf{J}^{2}, \\
\operatorname{Range}(\mathbf{J})=\operatorname{span}\left\{x^{[1]}, \ldots, \chi^{[M]}\right\}=\operatorname{Null}(\mathbf{I}+\mathbf{N}), \\
\operatorname{Null}(\mathbf{J})=\left(\operatorname{span}\left\{x^{[1]}, \ldots, x^{[M]}\right\}\right)^{\perp}=\operatorname{Null}(\mathbf{I}+\mathbf{N})^{\perp}=\operatorname{Range}\left(\mathbf{I}+\mathbf{N}^{*}\right) .
\end{gathered}
$$

Hence, we have $\mathbf{N J}=-\mathbf{J}$ and $\mathbf{J N}^{*}=\mathbf{J}^{*} \mathbf{N}^{*}=(\mathbf{N J})^{*}=-\mathbf{J}$. By multiplying (6.17) by $\mathbf{J}$, we obtain

$$
\mathbf{J} v=0, \quad\left(\mathbf{I}+\mathbf{N}^{*}\right) v=0
$$

Thus,

$$
v \in \operatorname{Null}(\mathbf{J}) \cap \operatorname{Null}\left(\mathbf{I}+\mathbf{N}^{*}\right)=\operatorname{Range}\left(\mathbf{I}+\mathbf{N}^{*}\right) \cap \operatorname{Null}\left(\mathbf{I}+\mathbf{N}^{*}\right) .
$$

Since $\widehat{A}=1$, thus the index of the function $\widehat{A}$ is given by (see [18] for the definition of the index)

$$
\kappa_{j}=0, \quad j=0,1, \ldots, m, \kappa=0 .
$$

The space $S^{+}$defined in [18, Equation (30)] is then given by $S^{+}=\operatorname{span}\{1\}$. Then, it follows from [18, Equation (92)] that the dimension of the space $\widetilde{S}^{+}$defined in [18, Equation (32)] is given by $\operatorname{dim}\left(\widetilde{S}^{+}\right)=M$. Similarly, it follows from [18, Equation (105)] that

$$
\operatorname{dim}\left(\operatorname{Null}\left(\mathbf{I}+\mathbf{N}^{*}\right)\right)=\operatorname{dim}(\operatorname{Null}(\mathbf{I}-\tilde{\mathbf{N}}))=M
$$

Thus, it follows from [18, Lemma 20(b)] that $\operatorname{Null}\left(\mathbf{I}+\mathbf{N}^{*}\right)=\widetilde{S}^{+}$and the space $\widetilde{R}^{+} \cap \widetilde{S}^{-}$in $[18$, Lemma 20(a)] contains only the zero function, that is, $\widetilde{R}^{+} \cap \widetilde{S}^{-}=\{0\}$. Thus, it follows from 
[18, Equation (103)] (applied to the adjoint function $\widetilde{A}(t)=\widehat{A}(t) / z^{\prime}(t)$ instead of $\left.\widehat{A}(t)\right)$ and from [18, Equation (100)] that

$$
\operatorname{Range}\left(\mathbf{I}+\mathbf{N}^{*}\right) \cap \operatorname{Null}\left(\mathbf{I}+\mathbf{N}^{*}\right)=\{0\} .
$$

Hence, it follows from (6.20) that $v=0$.

By solving the integral equation (6.15), we get $\theta_{p}(t)$. And solving the integral equation (5.7), we get $\phi^{[j]}, j=0,1, \ldots, M$, which gives $h_{j}$ through (5.6) which in turn gives $\mu_{j}$ through (5.8). By solving integral equation (4.8), (4.10), and (4.11) with the known values of $\mu_{j}$, we get $F(z)$. From the definition of $F(z)$, we get

$$
f^{\prime}(z(t))=\frac{F(z(t))}{z^{2}(t) z^{\prime}(t)} .
$$

Finally, from (3.14) and (6.24), the approximate boundary value of $f(z)$ is given by

$$
f(z)=\frac{|f(z)|}{i} T(z) \frac{\left|\theta_{p}^{\prime}(t)\right|}{\left|\theta_{p}^{\prime}(t)\right|} \frac{f^{\prime}(z)}{\left|f^{\prime}(z)\right|}, \quad z \in \Gamma .
$$

The approximate interior value of the function $f(z)$ is calculated by the Cauchy integral formula

$$
f(z)=\frac{a-z}{a z} \frac{1}{2 \pi i} \int_{\Gamma} \frac{a w f(w)}{a-w} \frac{1}{w-z} d w, \quad z \in \Gamma
$$

For points $z$ which are not close to the boundary, the integral in (6.26) is approximated by the trapezoidal rule. However, for the points $z$ closed to the boundary $\Gamma$, the numerical integration in (6.26) is nearly singular. This difficulty is overcome by using the fact that $(1 / 2 \pi i) \int_{\Gamma}(1 /(w-z)) d w=1$, and rewrite $f(z)$ as

$$
f(z)=\frac{((a-z) / a z)(1 / 2 \pi i) \int_{\Gamma}(a w f(w) /(a-w))(1 /(w-z)) d w}{\int_{\Gamma}(1 /(w-z)) d w}, \quad z \in \Omega .
$$

This idea has the advantage that the denominator in this formula compensates for the error in the numerator (see [23]). The integrals in (6.27) are approximated by the trapezoidal rule.

\section{Numerical Examples}

Since the function $z_{p}(t)$ is $2 \pi$-periodic, a reliable procedure for solving the integral equations (6.15), (5.7), and (4.8) with the conditions (4.10) and (4.11) numerically is by using the Nyström's method with the trapezoidal rule [24]. The trapezoidal rule is the most accurate method for integrating periodic functions numerically [25, page 134-142]. Thus, solving the integral equations numerically reduces to solving linear systems of the form

$$
A X=B
$$


Table 1: Error norm (unit circle).

\begin{tabular}{lll}
\hline$n$ & $\left\|\mu-\mu_{n}\right\|_{\infty}$ & $\left\|f-f_{n}(t)\right\|_{\infty}$ \\
\hline 8 & $1.8 \times 10^{-05}$ & $2.2 \times 10^{-02}$ \\
16 & $3.7 \times 10^{-10}$ & $5.0 \times 10^{-06}$ \\
32 & $8.8 \times 10^{-16}$ & $3.4 \times 10^{-14}$ \\
\hline
\end{tabular}

Table 2: The numerical values of $\mu_{0}$ for Example 7.2.

\begin{tabular}{cc}
\hline$n$ & $\mu_{0}$ \\
\hline 16 & 3.5383174719052 \\
32 & 3.5355590602433 \\
64 & 3.5355585660566 \\
128 & - \\
\hline
\end{tabular}

The above linear system (7.1) is uniquely solvable for sufficiently large number of collocation points on each boundary component, since the integral equations (6.15), (5.7), and (4.8) with the conditions (4.10) and (4.11) are uniquely solvable [26]. The computational details are similar to $[6,11-13]$.

\subsection{Regions of Connectivity One}

For numerical experiments, we have used some test regions of connectivity two, three, four, and five based on the examples given in $[2,4,7,12,13,15,27-29]$. All the computations were done using MATLAB 7.8.0.347(R2009a)(double precision floating point number). The number of points used in the discretization of each boundary component $\Gamma_{j}$ is $n$.

In this section, we have used three test regions of connectivity one. Only the first test region has known exact mapping function. The results for sup norm error between the exact values of $f, \mu_{1}$ and approximate values $f_{n}, \mu_{1 n}$ are shown in Table 1 .

Example 7.1. Consider a region $\Omega$ bounded by the unit circle

$$
\Gamma:\left\{z(t)=e^{i t}\right\}, \quad a=-0.2+0.2 i,
$$

Then, the exact mapping function is given by [1, page 340]

$$
g(z)=\frac{(a-z)}{a z(1-\bar{a} z)}, \quad r=\frac{1}{|a|}
$$

Figure 2 shows the region and its image based on our method. See Table 1 for results.

Example 7.2. Consider the elliptical region bounded by the ellipse

$$
\Gamma:\{z(t)=4 \cos t+2 i \sin t\}, \quad a=-0.2-0.2 i \text {. }
$$

Figure 3 shows the region and its image based on our method. See Table 2 for our computed value of $\mu_{0}$. 
Table 3: Error norm for Example 7.3.

\begin{tabular}{cc}
\hline$n$ & $\left\|\mu_{0}-\mu_{0 n}\right\|_{\infty}$ \\
\hline 8 & $1.0 \times 10^{-02}$ \\
16 & $7.2 \times 10^{-05}$ \\
32 & $1.1 \times 10^{-08}$ \\
64 & $4.6 \times 10^{-15}$ \\
\hline
\end{tabular}

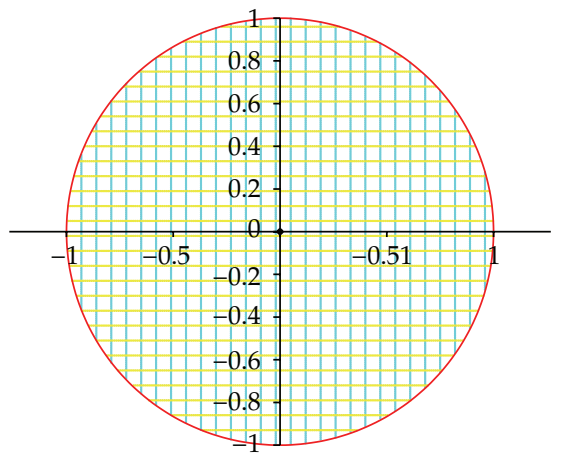

(a)

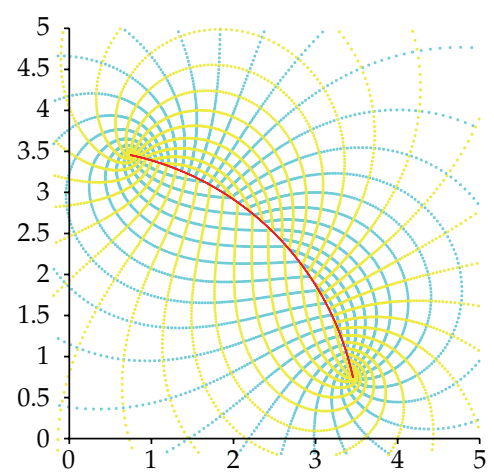

(b)

Figure 2: Mapping a region $\Omega$ bounded by unit circle onto a circular slit region.

Example 7.3. Consider a region $\Omega$ bounded by

$$
\Gamma:\left\{z(t)=(10+3 \cos 3 t) e^{i t}\right\}, \quad a=0.1-0.6 i .
$$

Figure 4 shows the region and its image based on our method. See Table 3 for comparison between our computed values of $\mu_{0}$ with those computed values $\mu_{0 n}$ of Nasser $[12,13]$.

\subsection{Regions of Connectivity Two}

In this section, we have used two test regions of connectivity two whose exact mapping functions are unknown. The first and second test regions are circular frame, and the third test region is bounded by an ellipse and circle. Figures 5-7 show the region and its image based on our method, and approximate values of $\mu_{0}$ and $\mu_{1}$ are shown in Tables 4-6.

Example 7.4 (circular frame). Consider a pair of circles [28]

$$
\begin{gathered}
\Gamma_{0}:\left\{z(t)=e^{i t}\right\}, \\
\Gamma_{1}:\left\{z(t)=-0.6+0.2 e^{-i t}\right\}, \quad t: 0 \leq t \leq 2 \pi, a=0.25+0.25 i,
\end{gathered}
$$

such that the region bounded by $\Gamma_{0}$ and $\Gamma_{1}$ is the region between a unit circle and a circle centered at -0.6 with radius 0.2 . Then, Figure 5 shows the region and its image based on our 


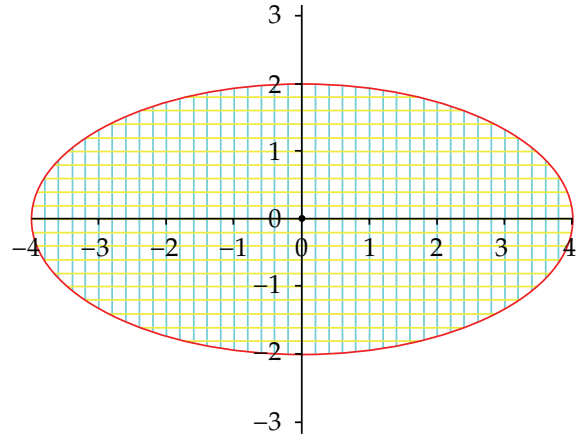

(a)

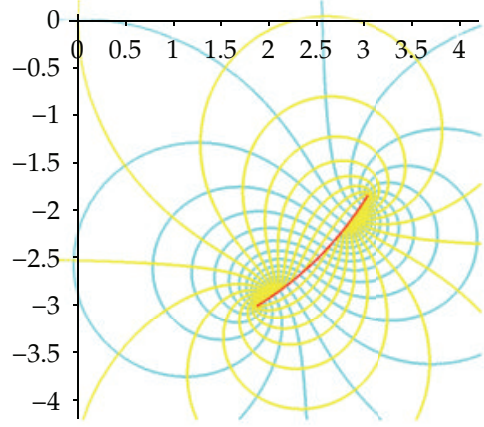

(b)

Figure 3: Mapping for Example 7.2.

Table 4: Error norm for Example 7.4.

\begin{tabular}{lll}
\hline $2 n$ & $\left\|\mu_{0}-\mu_{0 n}\right\|_{\infty}$ & $\left\|\mu_{1}-\mu_{1 n}\right\|_{\infty}$ \\
\hline 32 & $3.2 \times 10^{-03}$ & $5.8 \times 10^{-03}$ \\
64 & $2.4 \times 10^{-06}$ & $5.1 \times 10^{-06}$ \\
128 & $1.7 \times 10^{-12}$ & $3.5 \times 10^{-12}$ \\
256 & $8.8 \times 10^{-16}$ & $2.2 \times 10^{-15}$ \\
\hline
\end{tabular}

method. See Table 4 for comparison between our computed values of $\mu_{0}$ and $\mu_{1}$ with those computed values $\mu_{0 n}$ and $\mu_{1 n}$ of Nasser $[12,13]$.

Example 7.5 (ellipse with one circle). Consider a region $\Omega$ bounded by an ellipse and a circle

$$
\begin{gathered}
\Gamma_{0}:\{z(t)=4 \cos t+i \sin t\}, \\
\Gamma_{1}:\left\{z(t)=-1+0.25 e^{-i t}\right\}, \quad t: 0 \leq t \leq 2 \pi, a=-1.4,
\end{gathered}
$$

such that the region bounded by $\Gamma_{0}$ and $\Gamma_{1}$ is the region between an ellipse and a circle centered at -1 with radius 0.25 . Then, Figure 6 shows the region and its image based on our method. See Table 5 for comparison between our computed values of $\mu_{0}$ and $\mu_{1}$ with those computed values $\mu_{0 n}$ and $\mu_{1 n}$ of Nasser $[12,13]$.

Example 7.6 (two ellipses). Consider a region $\Omega$ bounded by pair of ellipses

$$
\begin{gathered}
\Gamma_{0}:\{z(t)=4 \cos t+i \sin t\}, \\
\Gamma_{1}:\{z(t)=1+0.7 \cos t-0.3 i \sin t\}, \quad t: 0 \leq t \leq 2 \pi, a=2.3 .
\end{gathered}
$$

Figure 7 shows the region and its image based on our method. See Table 6 for comparison between our computed values of $\mu_{0}$ and $\mu_{1}$ with those computed values $\mu_{0 n}$ and $\mu_{1 n}$ of Nasser $[12,13]$. 


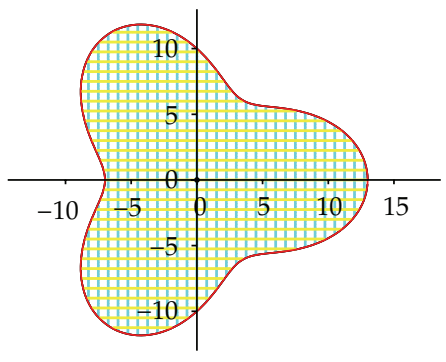

(a)

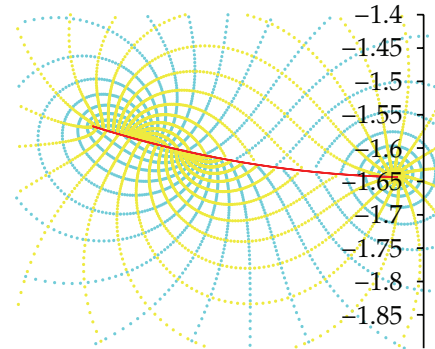

(b)

Figure 4: Mapping an original region and its image.

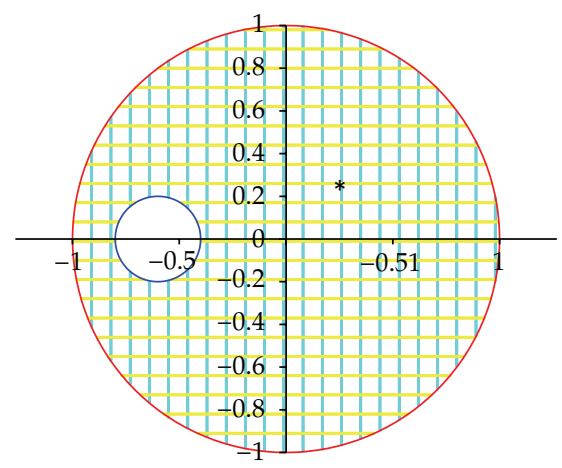

(a)

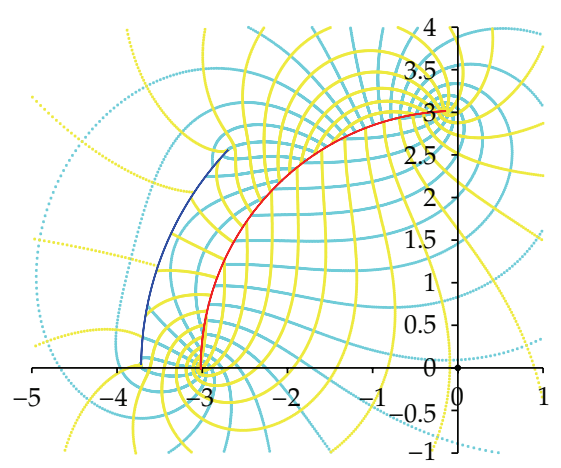

(b)

Figure 5: Mapping a region $\Omega$ bounded by two circles onto a circular slit region.

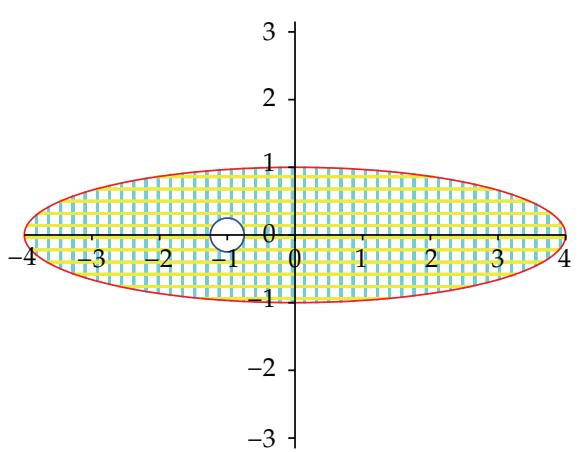

(a)

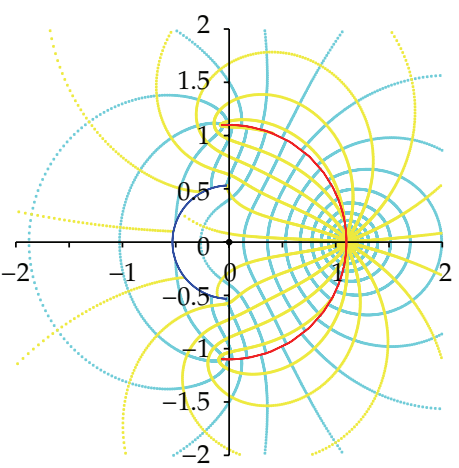

(b)

Figure 6: Mapping a region $\Omega$ bounded by an ellipse and a circle onto a circular slit region.

Table 5: Error norm for Example 7.5.

\begin{tabular}{lll}
\hline $2 n$ & $\left\|\mu_{0}-\mu_{0 n}\right\|_{\infty}$ & $\left\|\mu_{1}-\mu_{1 n}\right\|_{\infty}$ \\
\hline 64 & $1.5 \times 10^{-03}$ & $6.2 \times 10^{-04}$ \\
128 & $4.9 \times 10^{-07}$ & $8.5 \times 10^{-10}$ \\
256 & $7.1 \times 10^{-14}$ & $3.5 \times 10^{-14}$ \\
\hline
\end{tabular}




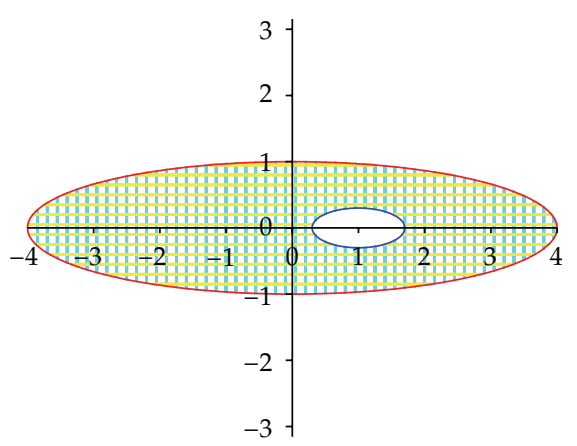

(a)

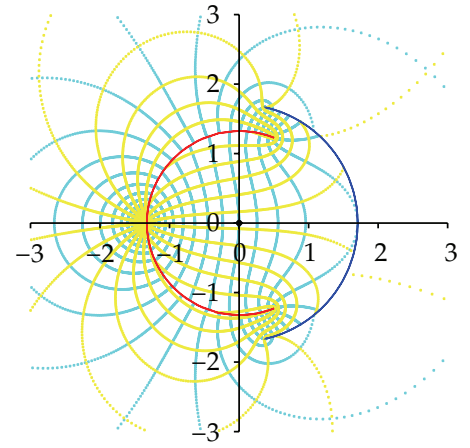

(b)

Figure 7: Mapping a region $\Omega$ bounded by two ellipses onto a circular slit region.

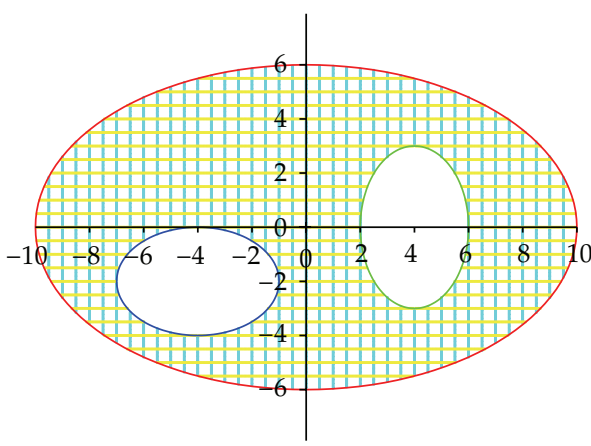

(a)

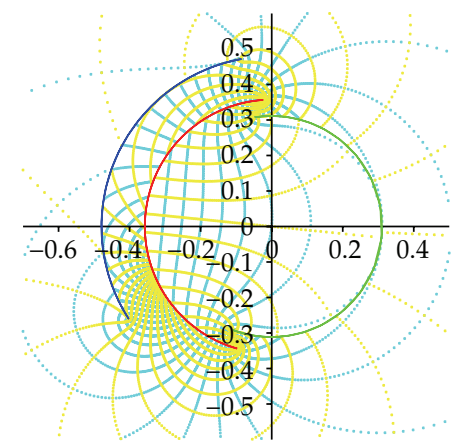

(b)

Figure 8: Mapping a region $\Omega$ bounded by three ellipses onto a circular slit region.

\subsection{Regions of Connectivity Three}

In this section, we have used three test regions of connectivity three. The first test region is bounded by three ellipses, the second test region is bounded by an ellipse and two circles, and the third test region is a circular region. The results for sup norm error between the our numerical values of $\mu_{0}, \mu_{1}, \mu_{2}$ and the computed values of $\mu_{0 n}, \mu_{1 n}, \mu_{2 n}$ obtained from $[12,13]$ are shown in Tables 7-9.

Example 7.7 (three ellipses). Let $\Omega$ be the region bounded by

$$
\begin{gathered}
\Gamma_{0}:\{z(t)=10 \cos t+6 i \sin t\}, \\
\Gamma_{1}:\{z(t)=-4-2 i+3 \cos t-2 i \sin t\}, \\
\Gamma_{2}:\{z(t)=4+2 \cos t-3 i \sin t\}, \quad 0 \leq t \leq 2 \pi, a=7 .
\end{gathered}
$$

Figure 8 shows the region and its image based on our method. See Table 7 for comparison between our computed values of $\mu_{0}, \mu_{1}$, and $\mu_{2}$ with those computed values of Nasser [12]. 


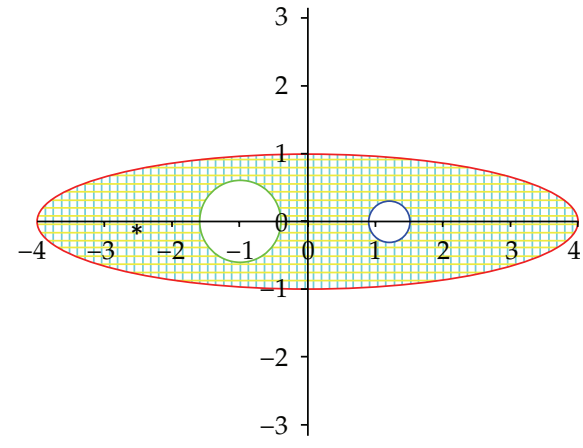

(a)

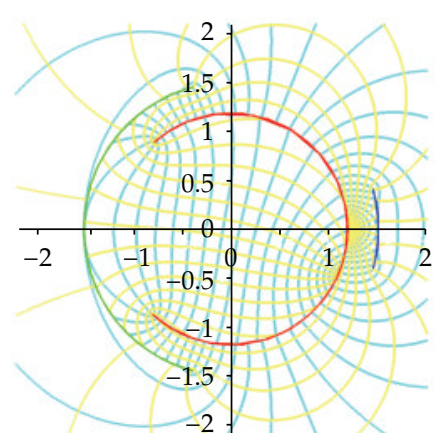

(b)

Figure 9: Mapping a region $\Omega$ bounded by an ellipse and two circles onto a circular slit region.

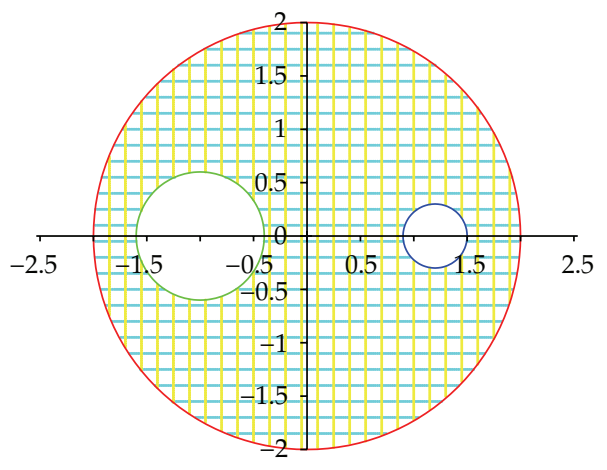

(a)

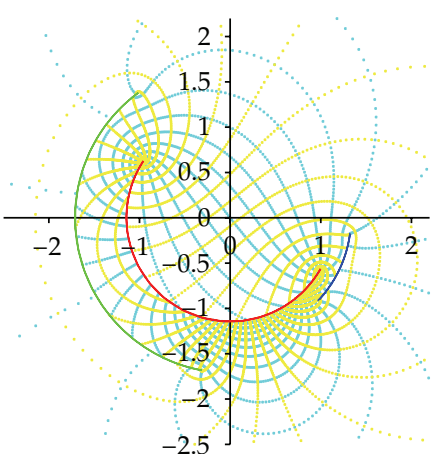

(b)

Figure 10: Mapping a region $\Omega$ bounded by three circles onto a circular slit region.

Example 7.8 (ellipse with two circles). Let $\Omega$ be the region bounded by $[7,13,15]$

$$
\begin{gathered}
\Gamma_{0}:\{z(t)=4 \cos t+i \sin t\}, \\
\Gamma_{1}:\{z(t)=1.2+0.3(\cos t-i \sin t)\}, \\
\Gamma_{2}:\{z(t)=-1+0.6(\cos t-i \sin t)\}, \quad 0 \leq t \leq 2 \pi, a=-2.5-0.1 i
\end{gathered}
$$

Figure 9 shows the region and its image based on our method. See Table 8 for comparison between our computed values of $\mu_{0}, \mu_{1}$, and $\mu_{2}$ with those computed values of Nasser [13].

Example 7.9 (three circles). Let $\Omega$ be the region bounded by

$$
\begin{gathered}
\Gamma_{0}:\left\{z(t)=2 e^{i t}\right\}, \\
\Gamma_{1}:\left\{z(t)=1.2+0.3 e^{-i t}\right\}, \\
\Gamma_{2}:\left\{z(t)=-1+0.6 e^{-i t}\right\}, \quad 0 \leq t \leq 2 \pi, a=0.5-1.25 i .
\end{gathered}
$$




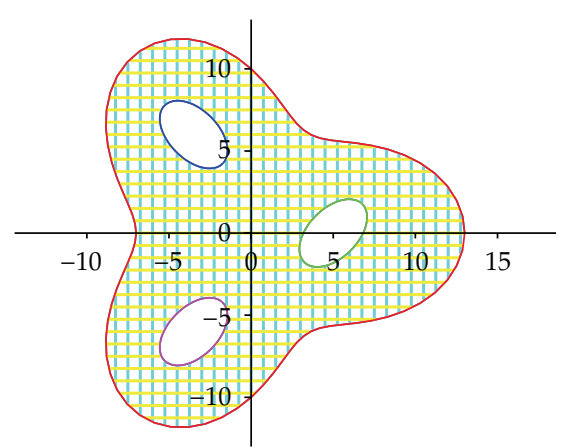

(a)

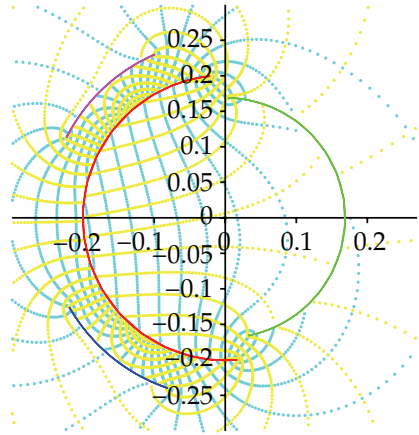

(b)

Figure 11: Mapping for Example 7.10.

Table 6: Error norm for Example 7.6.

\begin{tabular}{lll}
\hline $2 n$ & $\left\|\mu_{0}-\mu_{0 n}\right\|_{\infty}$ & $\left\|\mu_{1}-\mu_{1 n}\right\|_{\infty}$ \\
\hline 64 & $2.3 \times 10^{-03}$ & $2.4 \times 10^{-03}$ \\
128 & $7.4 \times 10^{-07}$ & $9.5 \times 10^{-07}$ \\
256 & $7.3 \times 10^{-14}$ & $9.9 \times 10^{-14}$ \\
\hline
\end{tabular}

Table 7: Error norm for Example 7.7.

\begin{tabular}{llll}
\hline $3 n$ & $\left\|\mu_{0}-\mu_{0 n}\right\|_{\infty}$ & $\left\|\mu_{1}-\mu_{1 n}\right\|_{\infty}$ & $\left\|\mu_{2}-\mu_{2 n}\right\|_{\infty}$ \\
\hline 48 & $5.1 \times 10^{-04}$ & $1.3 \times 10^{-03}$ & $4.7 \times 10^{-04}$ \\
96 & $2.8 \times 10^{-06}$ & $7.5 \times 10^{-06}$ & $3.9 \times 10^{-06}$ \\
192 & $2.4 \times 10^{-10}$ & $6.3 \times 10^{-10}$ & $3.1 \times 10^{-10}$ \\
384 & $5.5 \times 10^{-17}$ & $2.7 \times 10^{-16}$ & $4.9 \times 10^{-16}$ \\
\hline
\end{tabular}

Table 8: Error norm for Example 7.8.

\begin{tabular}{llll}
\hline $3 n$ & $\left\|\mu_{0}-\mu_{0 n}\right\|_{\infty}$ & $\left\|\mu_{1}-\mu_{1 n}\right\|_{\infty}$ & $\left\|\mu_{2}-\mu_{2 n}\right\|_{\infty}$ \\
\hline 96 & $1.6 \times 10^{-05}$ & $1.0 \times 10^{-03}$ & $4.9 \times 10^{-03}$ \\
192 & $2.7 \times 10^{-06}$ & $2.8 \times 10^{-06}$ & $8.6 \times 10^{-07}$ \\
384 & $1.2 \times 10^{-11}$ & $1.4 \times 10^{-11}$ & $1.2 \times 10^{-11}$ \\
\hline
\end{tabular}

Table 9: The numerical values of $\mu_{0}, \mu_{1}$, and $\mu_{2}$ for Example 7.9.

\begin{tabular}{lccc}
\hline $3 n$ & $\mu_{0}$ & $\mu_{1}$ & $\mu_{2}$ \\
\hline 96 & 1.144844712112 & 1.333447560114 & 1.711779222648 \\
192 & 1.144844080644 & 1.333446944282 & 1.711778670173 \\
384 & - & 1.333446944281 & - \\
\hline
\end{tabular}

Table 10: Error norm for Example 7.10.

\begin{tabular}{lllll}
\hline $4 n$ & $\left\|\mu_{0}-\mu_{0 n}\right\|_{\infty}$ & $\left\|\mu_{1}-\mu_{1 n}\right\|_{\infty}$ & $\left\|\mu_{2}-\mu_{2 n}\right\|_{\infty}$ & $\left\|\mu_{3}-\mu_{3 n}\right\|_{\infty}$ \\
\hline 64 & $6.7 \times 10^{-05}$ & $7.2 \times 10^{-05}$ & $9.9 \times 10^{-05}$ & $2.2 \times 10^{-05}$ \\
128 & $6.4 \times 10^{-09}$ & $5.0 \times 10^{-08}$ & $1.8 \times 10^{-09}$ & $4.5 \times 10^{-08}$ \\
256 & $6.8 \times 10^{-13}$ & $1.0 \times 10^{-12}$ & $9.8 \times 10^{-13}$ & $9.7 \times 10^{-13}$ \\
512 & $1.3 \times 10^{-16}$ & $1.2 \times 10^{-15}$ & $3.0 \times 10^{-16}$ & $4.4 \times 10^{-16}$ \\
\hline
\end{tabular}


Table 11: The numerical values of $\mu_{0}, \mu_{1}, \mu_{2}$, and $\mu_{3}$ for Example 7.11.

\begin{tabular}{lcccc}
\hline $4 n$ & $\mu_{0}$ & $\mu_{1}$ & $\mu_{2}$ & $\mu_{3}$ \\
\hline 64 & 2.97316998311 & 2.50170500411 & 3.45373711618 & 3.69125205510 \\
128 & 2.96757277502 & 2.49923061605 & 3.45041067650 & 3.69904161729 \\
256 & 2.96756361086 & 2.49922735100 & 3.45040617845 & 3.69905124306 \\
512 & 2.96756361085 & 2.49922735099 & 3.45040617844 & 3.69905124308 \\
\hline
\end{tabular}

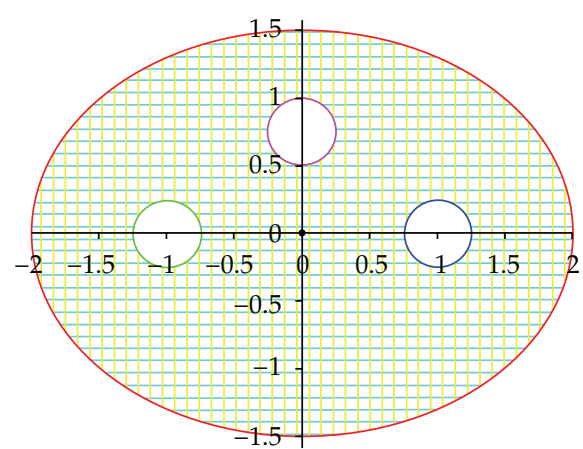

(a)

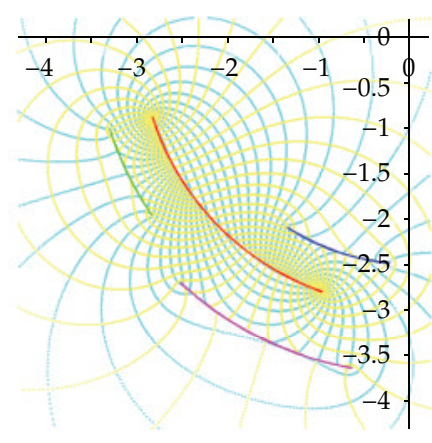

(b)

Figure 12: Mapping a region $\Omega$ bounded by an ellipse and three circles onto a circular slit region.

Figure 10 shows the region and its image based on our method. See Table 9 for our computed values of $\mu_{0}, \mu_{1}$, and $\mu_{2}$.

\subsection{Regions of Connectivity Four and Five}

In this section, we have used four test regions for multiply connected regions whose exact mapping functions are unknown. The results for sup norm error for first and third regions between the our numerical values of $\mu_{0}, \mu_{1}, \mu_{2}, \mu_{3}, \mu_{4}$ and the computed values of $\mu_{0 n}, \mu_{1 n}$, $\mu_{2 n}, \mu_{3 n}, \mu_{4 n}$ obtained from [12] are shown in Tables 10 and 12.

Example 7.10. Let $\Omega$ be the region bounded by [12]

$$
\begin{gathered}
\Gamma_{0}:\left\{z(t)=(10+3 \cos 3 t) e^{i t}\right\}, \\
\Gamma_{1}:\left\{z(t)=-3.5+6 i+0.5 e^{-i \pi / 4}\left(e^{i t}+4 e^{-i t}\right)\right\}, \\
\Gamma_{2}:\left\{z(t)=5+0.5 e^{i \pi / 4}\left(e^{i t}+4 e^{-i t}\right)\right\}, \\
\Gamma_{3}:\left\{z(t)=-3.5-6 i+0.5 e^{i \pi / 4}\left(e^{i t}+4 e^{-i t}\right)\right\}, \quad 0 \leq t \leq 2 \pi, a=8.5+0.1 i .
\end{gathered}
$$

Figure 11 shows the region and its image based on our method. See Table 10 for comparison between our computed values of $\mu_{0}, \mu_{1}, \mu_{2}$, and $\mu_{3}$ with those computed values of Nasser [12]. 


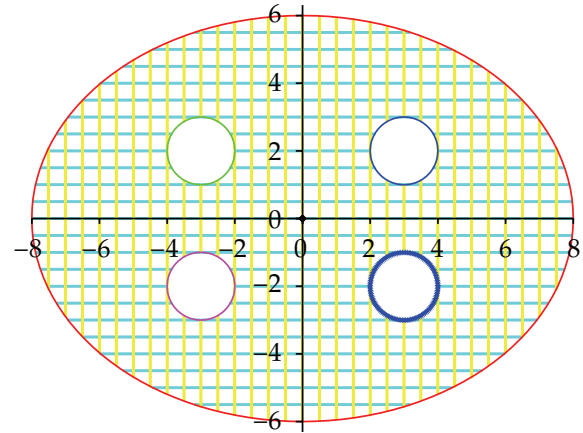

(a)

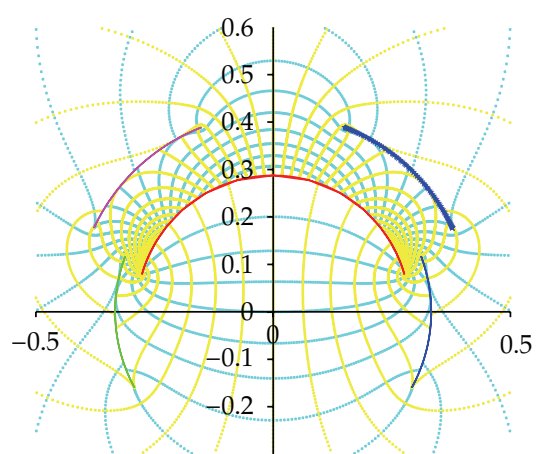

(b)

Figure 13: Mapping a region $\Omega$ bounded by an ellipse and four circles onto a circular slit region.

Table 12: Error norm for Example 7.12.

\begin{tabular}{lccccc}
\hline $5 n$ & $\left\|\mu_{0}-\mu_{0 n}\right\|_{\infty}$ & $\left\|\mu_{1}-\mu_{1 n}\right\|_{\infty}$ & $\left\|\mu_{2}-\mu_{2 n}\right\|_{\infty}$ & $\left\|\mu_{3}-\mu_{3 n}\right\|_{\infty}$ & $\left\|\mu_{4}-\mu_{4 n}\right\|_{\infty}$ \\
\hline 80 & $4.2 \times 10^{-05}$ & $4.5 \times 10^{-05}$ & $4.5 \times 10^{-05}$ & $4.4 \times 10^{-05}$ & $4.3 \times 10^{-05}$ \\
160 & $1.1 \times 10^{-07}$ & $3.2 \times 10^{-08}$ & $3.2 \times 10^{-08}$ & $6.6 \times 10^{-08}$ & $6.6 \times 10^{-08}$ \\
320 & $1.6 \times 10^{-13}$ & $5.7 \times 10^{-14}$ & $5.7 \times 10^{-14}$ & $1.2 \times 10^{-13}$ & $1.2 \times 10^{-13}$ \\
400 & $9.9 \times 10^{-16}$ & 0 & $9.9 \times 10^{-16}$ & 0 & 0 \\
\hline
\end{tabular}

Example 7.11 (ellipse with three circles). Let $\Omega$ be the region bounded by

$$
\begin{gathered}
\Gamma_{0}:\{z(t)=2 \cos t+1.5 i \sin t\}, \\
\Gamma_{1}:\{z(t)=1+0.25(\cos t-i \sin t)\}, \\
\Gamma_{2}:\{z(t)=-1+0.25(\cos t-i \sin t)\}, \\
\Gamma_{3}:\{z(t)=0.75 i+0.25(\cos t-i \sin t)\}, \quad 0 \leq t \leq 2 \pi, a=0.25-0.25 i .
\end{gathered}
$$

Figure 12 shows the region and its image based on our method. See Table 11 for our computed values of $\mu_{0}, \mu_{1}, \mu_{2}$, and $\mu_{3}$.

Example 7.12 (ellipse with four circles). Let $\Omega$ be the region bounded by

$$
\begin{gathered}
\Gamma_{0}:\{z(t)=0.2+8 \cos t+6 i \sin t\}, \\
\Gamma_{1}:\{z(t)=3+2 i+\cos t-i \sin t\}, \\
\Gamma_{2}:\{z(t)=-3+2 i+\cos t-i \sin t\}, \\
\Gamma_{3}:\{z(t)=-3-2 i+\cos t-i \sin t\}, \\
\Gamma_{4}:\{z(t)=3-2 i+\cos t-i \sin t\}, \quad 0 \leq t \leq 2 \pi, a=4 i .
\end{gathered}
$$




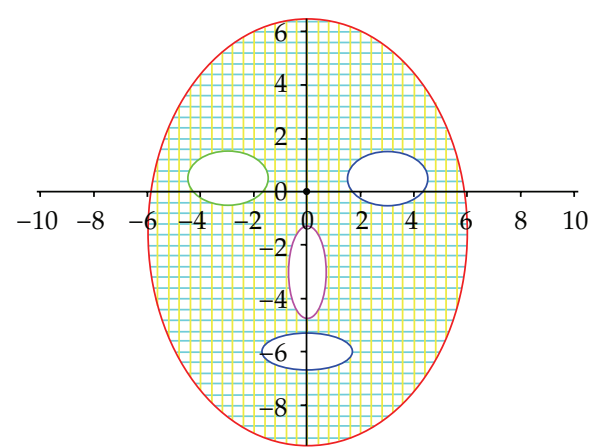

(a)

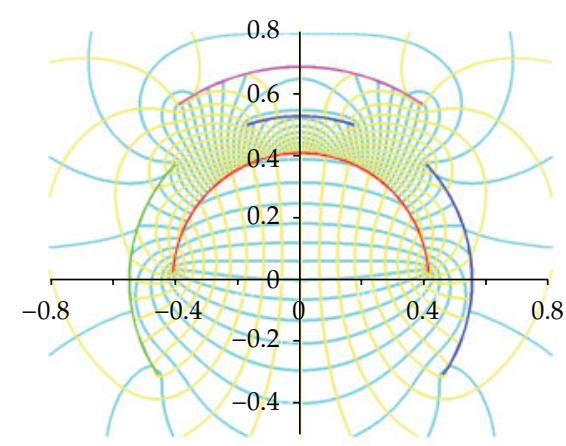

(b)

Figure 14: Mapping a region $\Omega$ bounded by five ellipses onto a circular slit region.

Table 13: The numerical values of $\mu_{0}, \mu_{1}, \mu_{2}, \mu_{3}$, and $\mu_{4}$ for Example 7.13.

\begin{tabular}{lccccc}
\hline $5 n$ & $\mu_{0}$ & $\mu_{1}$ & $\mu_{2}$ & $\mu_{3}$ & $\mu_{4}$ \\
\hline 160 & 0.4081769461 & 0.5470254751 & 0.5470254751 & 0.6850879289 & 0.5258641902 \\
320 & 0.4081097591 & 0.5470505181 & 0.5470505181 & 0.6850466360 & 0.5258066821 \\
400 & 0.4081097885 & 0.5470505071 & 0.5470505071 & 0.6850466537 & 0.5258067072 \\
\hline
\end{tabular}

Figure 13 shows the region and its image based on our method. See Table 12 for comparison between our computed values of $\mu_{0}, \mu_{1}, \mu_{2}, \mu_{3}$, and $\mu_{4}$ with those computed values of Nasser [12].

Example 7.13 (five ellipses). Let $\Omega$ be the region bounded by

$$
\begin{gathered}
\Gamma_{0}:\{z(t)=-1.5 i+6 \cos t+8 i \sin t\}, \\
\Gamma_{1}:\{z(t)=3+0.5 i+1.5 \cos t-i \sin t\}, \\
\Gamma_{2}:\{z(t)=-3+0.5 i+1.5 \cos t-i \sin t\}, \\
\Gamma_{3}:\{z(t)=-3 i+0.7 \cos t-1.7 i \sin t\}, \\
\Gamma_{4}:\{z(t)=-6 i+1.7 \cos t-0.7 i \sin t\}, \quad 0 \leq t \leq 2 \pi, a=0.4 i .
\end{gathered}
$$

Figure 14 shows the region and its image based on our method. See Table 13 for our computed values of $\mu_{0}, \mu_{1}, \mu_{2}, \mu_{3}$, and $\mu_{4}$.

\section{Conclusion}

In this paper, we have constructed new boundary integral equations for conformal mapping of multiply regions onto a circular slit region. We have also constructed a new method to find the values of modulus of $f(z)$. The advantage of our method is that our boundary integral equations are all linear. Several mappings of the test regions of connectivity one, two, three, four, and five were computed numerically using the proposed method. After the boundary values of the mapping function are computed, the interior mapping function is calculated by 
the means of Cauchy integral formula. The numerical examples presented have illustrated that our boundary integral equation method has high accuracy.

\section{Acknowledgments}

This work was supported in part by the Malaysian Ministry of Higher Education (MOHE) through the Research Management Centre (RMC), Universiti Teknologi Malaysia (FRGS Vote 78479). This support is gratefully acknowledged. The authors would like to thank an anonymous referee for careful reading of the paper and constructive comments and suggestions that substantially improved the presentation of the paper.

\section{References}

[1] Z. Nehari, Conformal Mapping, Dover Publication, New York, NY, USA, 1952.

[2] K. Amano, "A charge simulation method for the numerical conformal mapping of interior, exterior and doubly-connected domains," Journal of Computational and Applied Mathematics, vol. 53, no. 3, pp. 353-370, 1994.

[3] D. Crowdy and J. Marshall, "Conformal mappings between canonical multiply connected domains," Computational Methods and Function Theory, vol. 6, no. 1, pp. 59-76, 2006.

[4] S. W. Ellacott, "On the approximate conformal mapping of multiply connected domains," Numerische Mathematik, vol. 33, no. 4, pp. 437-446, 1979.

[5] P. Henrici, Applied and Computational Complex Analysis, vol. 3, John Wiley \& Sons, New York, NY, USA, 1974.

[6] L. N. Hu, Boundary integral equations approach for numerical conformal mapping of multiply connected regions, Ph.D. thesis, Department of Mathematics, Universiti Teknologi Malaysia, Johor, Malaysia, 2009.

[7] C. A. Kokkinos, N. Papamichael, and A. B. Sideridis, "An orthonormalization method for the approximate conformal mapping of multiply-connected domains," IMA Journal of Numerical Analysis, vol. 10, no. 3, pp. 343-359, 1990.

[8] P. K. Kythe, Computational Conformal Mapping, Birkhauser Boston, New Orleans, La, USA, 1998.

[9] A. H. M. Murid and M. R. M. Razali, "An integral equation method for conformal mapping of doubly connected regions," Matematika, vol. 15, no. 2, pp. 79-93, 1999.

[10] A. H. M. Murid and N. A. Mohamed, "Numerical conformal mapping of doubly connected regions via the Kerzman-Stein kernel," International Journal of Pure and Applied Mathematics, vol. 36, no. 2, pp. 229-250, 2007.

[11] A. H. M. Murid and L.-N. Hu, "Numerical experiment on conformal mapping of doubly connected regions onto a disk with a slit," International Journal of Pure and Applied Mathematics, vol. 51, no. 4, pp. 589-608, 2009.

[12] M. M. S. Nasser, "A boundary integral equation for conformal mapping of bounded multiply connected regions," Computational Methods and Function Theory, vol. 9, no. 1, pp. 127-143, 2009.

[13] M. M. S. Nasser, "Numerical conformal mapping via a boundary integral equation with the generalized Neumann kernel," SIAM Journal on Scientific Computing, vol. 31, no. 3, pp. 1695-1715, 2009.

[14] D. Okano, H. Ogata, K. Amano, and M. Sugihara, “Numerical conformal mappings of bounded multiply connected domains by the charge simulation method," Journal of Computational and Applied Mathematics, vol. 159, no. 1, pp. 109-117, 2003.

[15] L. Reichel, "A fast method for solving certain integral equations of the first kind with application to conformal mapping," Journal of Computational and Applied Mathematics, vol. 14, no. 1-2, pp. 125-142, 1986, Special issue on numerical conformal mappin.

[16] G. T. Symm, "Conformal mapping of doubly-connected domains," Numerische Mathematik, vol. 13, pp. 448-457, 1969.

[17] M. M. S. Nasser, "Numerical conformal mapping of multiply connected regions onto the second, third and fourth categories of Koebe's canonical slit domains," Journal of Mathematical Analysis and Applications, vol. 382, no. 1, pp. 47-56, 2011. 
[18] R. Wegmann and M. M. S. Nasser, "The Riemann-Hilbert problem and the generalized Neumann kernel on multiply connected regions," Journal of Computational and Applied Mathematics, vol. 214, no. 1, pp. 36-57, 2008.

[19] A. W. K. Sangawi, A. H. M. Murid, and M. M. S. Nasser, "Linear integral equations for conformal mapping of bounded multiply connected regions onto a disk with circular slits," Applied Mathematics and Computation, vol. 218, no. 5, pp. 2055-2068, 2011.

[20] M. M. S. Nasser, Boundary integral equation approach for Riemann problem, Ph.D. thesis, Department of Mathematics, Universiti Teknologi Malaysia, Johor, Malaysia, 2005.

[21] R. V. Churchill and J. W. Brown, Complex Variables and Applications, McGraw-Hill, New York, NY, USA, 1984.

[22] M. M. S. Nasser, A. H. M. Murid, M. Ismail, and E. M. A. Alejaily, “Boundary integral equations with the generalized Neumann kernel for Laplace's equation in multiply connected regions," Applied Mathematics and Computation, vol. 217, no. 9, pp. 4710-4727, 2011.

[23] J. Helsing and R. Ojala, "On the evaluation of layer potentials close to their sources," Journal of Computational Physics, vol. 227, no. 5, pp. 2899-2921, 2008.

[24] K. E. Atkinson, The Numerical Solution of Integral Equations of the Second Kind, Cambridge University Press, Cambridge, UK, 1997.

[25] P. J. Davis and P. Rabinowitz, Methods of Numerical Integration, Academic Press, Orlando, Fla, USA, 2nd edition, 1984.

[26] K. E. Atkinson, A Survey of Numerical Methods for the Solution of Fredholm Integral Equations of the Second Kind, Society for Industrial and Applied Mathematics, Philadelphia, Pa, USA, 1976.

[27] W. von Koppenfels and F. Stallmann, Praxis der Konformen Abbildung, Springer-Verlag, Berlin, Germany, 1959.

[28] E. B. Saff and A. D. Snider, Fundamentals of Complex Analysis, Pearson Education Inc., Upper Saddle River, NJ, USA, 2003.

[29] E. T. Whittaker and G. N. Watson, A Course of Modern Analysis, Cambridge University Press, Cambridge, UK, 1927. 


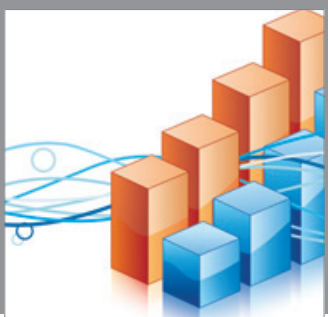

Advances in

Operations Research

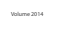

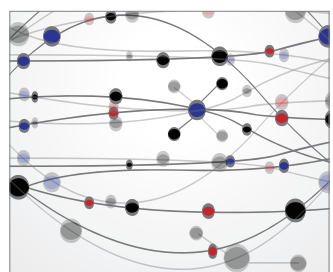

\section{The Scientific} World Journal
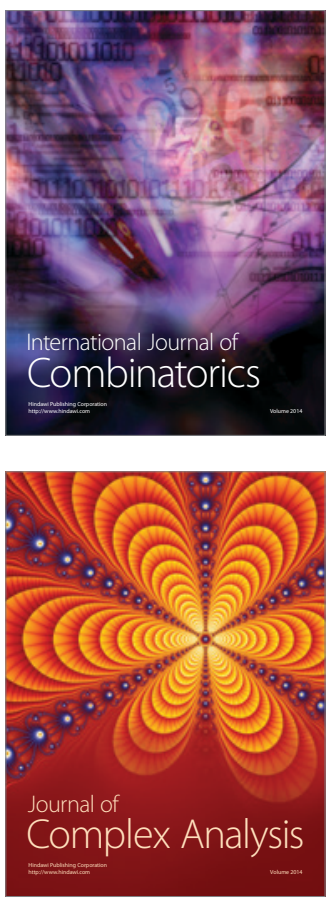

International Journal of

Mathematics and

Mathematical

Sciences
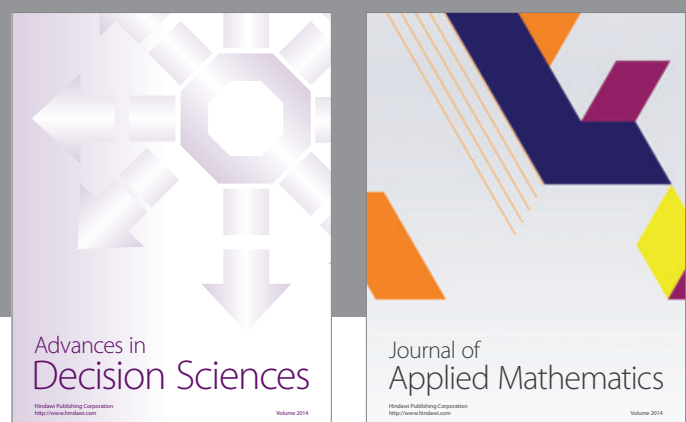

Journal of

Applied Mathematics
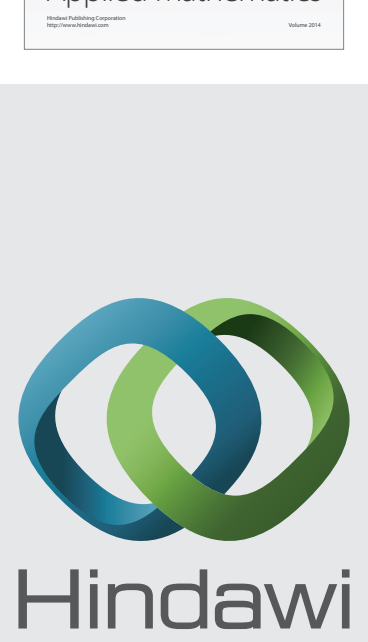

Submit your manuscripts at http://www.hindawi.com
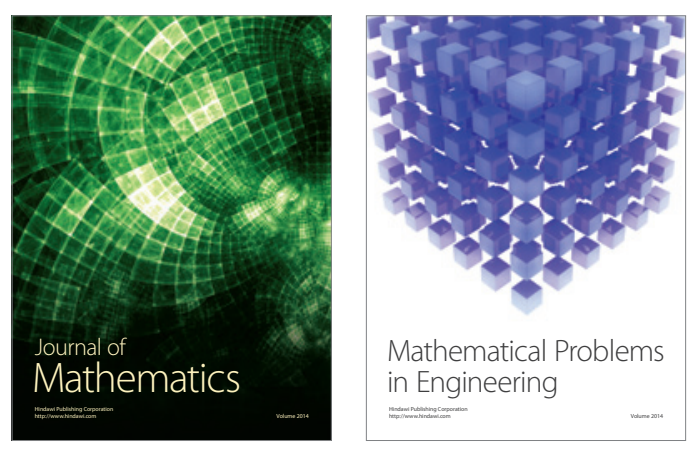

Mathematical Problems in Engineering
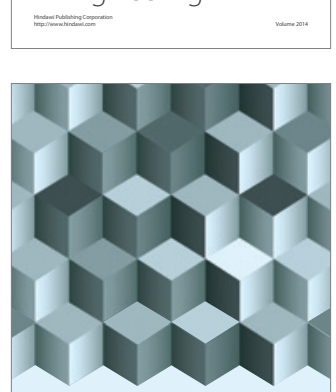

Journal of

Function Spaces
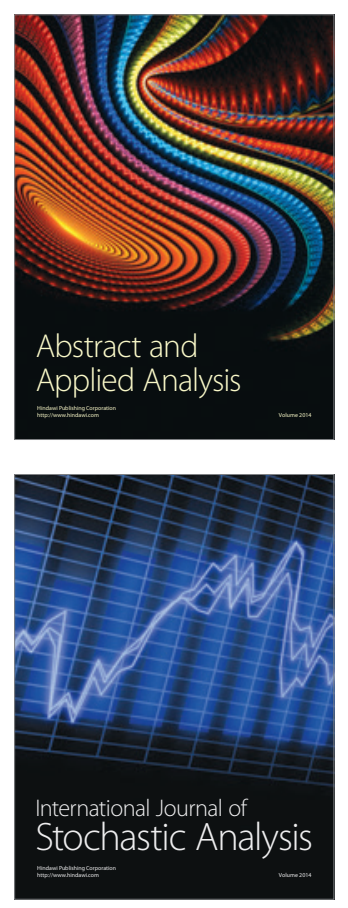

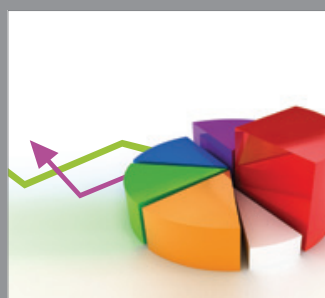

ournal of

Probability and Statistics

Promensencen
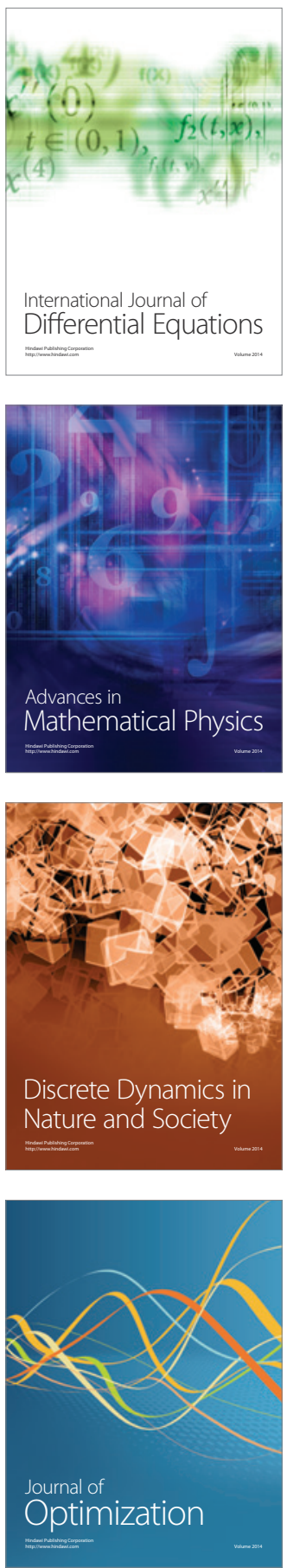